\title{
Design of an Array Antenna Hood for Jamming Simulator
}

\author{
Mi-Suk Kim ${ }^{\dagger}$, Jeong-Gab Ju, Jong Seong Kim, Seok Bo Son, Sang Jun Yun \\ NAVCOURS Co., Ltd, 66-6 Techno 2 Ro, Yuseong-gu, Daejeon 305-509, Korea
}

\begin{abstract}
In this study, due to the necessity of the equipment for inspecting an anti-jamming function depending on the expansion of the development of anti-jamming systems, an array antenna hood that can inspect the anti-jamming function of an antijamming system while being installed at the system was designed and manufactured. The manufactured array antenna hood plays a role in radiating GNSS signals and jamming signals. Based on an experiment, it was demonstrated that using a nearfield radiation method, the manufactured array antenna hood could be used for examining the normal operation of an antijamming system function in outdoor or indoor environments rather than an anechoic chamber.
\end{abstract}

Keywords: anti-jamming system, CRPA, near-field

\section{INTRODUCTION}

A satellite navigation system, which is represented by the Global Positioning System (GPS), is widely used for military purposes as well as civilian purposes. A satellite navigation system has the advantage of continuously providing precise navigation information. However, as a satellite navigation system uses the weak signals transmitted from satellites at an altitude of 20,200 km, it has the disadvantage of losing its function due to unintentional or intentional interference signals (Kim 2013). To effectively eliminate or suppress the interference and jamming signals that enter a satellite navigation receiver, diverse anti-jamming techniques have been studied. As a result, anti-jamming systems have been actively developed, and evaluation techniques for evaluating them also have come into request. However, these evaluation techniques require a lot of cost, time, and space (Bae et al. 2010). In this study, to overcome this disadvantage, an array antenna hood for inspecting the function of an anti-jamming system including array antenna was developed.

Received Mar 18, 2014 Revised Apr 30, 2014 Accepted May 11, 2014

†Corresponding Author

E-mail: gw4992@navcours.com

Tel: +82-42-363-9262 Fax: +82-42-363-9290

\section{ANTI-JAMMING TECHNIQUE AND EVALUATION TECHNIQUE}

\subsection{Anti-Jamming Technique}

Anti-jamming techniques for eliminating or suppressing the interference and jamming signals that enter a satellite navigation receiver system have been studied, and they are introduced in a number of papers. As shown in Fig. 1, antijamming techniques are presented as pre-correlation or post-correlation techniques based on the point at which signals are processed in a satellite navigation receiver. Applicable techniques are as follows (Bae et al. 2010).

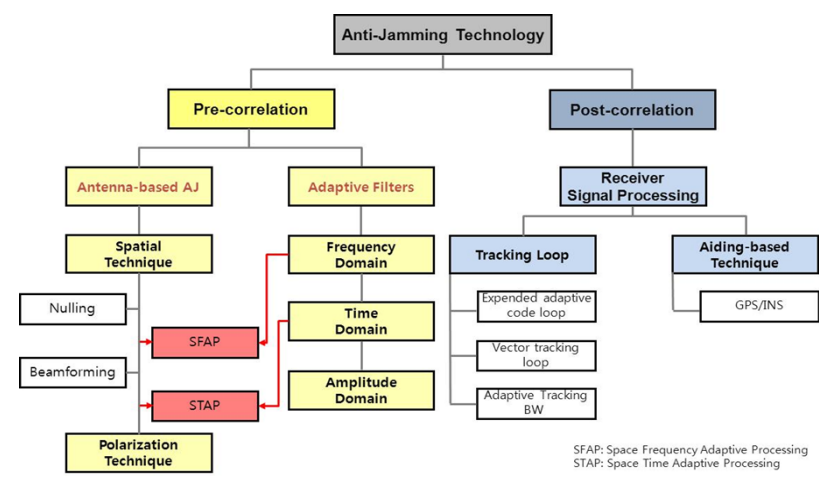

Fig. 1. Anti-jamming technique. 


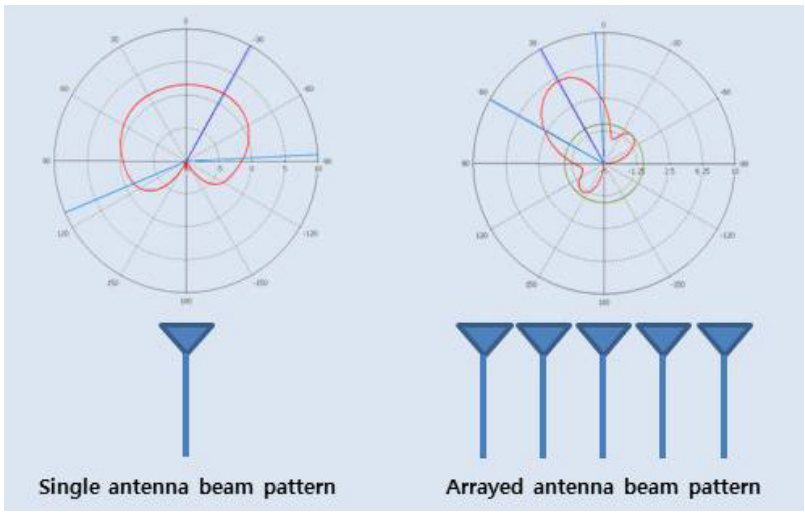

Fig. 2. Beam steered array.

\subsubsection{Antenna Technique}

The antenna technique is a technique that uses adaptive array antenna. It can be divided into a beam steered array technique that increases gain by forming narrow beamwidth in the satellite signal direction, and a null steered array technique that suppresses jamming signals by forming null in the jamming signal direction (Kim 2013). The beam steered array technique is a technique that forms narrow beamwidth in the satellite signal direction by arranging a number of antennas, as shown in Fig. 2. On the other hand, in the case of the Controlled Reception Pattern Antenna (CRPA) technique using the null steered array technique, nulling is performed by controlling the phase and gain of each antenna through obtaining jammer direction information (Maloney et al. 2007). If the number of arranged antennas is $\mathrm{N}$, it can form nulls for $\mathrm{N}-1$ jamming signals. Fig. 3 shows a method that deals with jamming signals using the null steered array technique. As shown in the figure, it suppresses jamming signals by forming nulls for the jamming signals that are entered in the jammer direction. The satellite signals in the direction other than that of the jamming signals can be effectively used. If the number of arranged antennas increases, the anti-jamming performance is improved because it can deal with a number of jammers. However, the size of the antenna increases, and the amount of signal processing calculation increases (Kim 2013).

\subsubsection{Adaptive Filtering Technique}

The filtering technique is a technique that blocks the jamming power that enters a receiver. It is a method that uses a band-pass filter with a sharp cut-off characteristic that suppresses the jamming signals in the band other than that of satellite navigation signals and passes only the satellite signals. It is vulnerable to the jamming signals whose band is identical to that of satellite signals (Kim 2013).

\subsubsection{Automatic Gain Control (AGC) Technique}

If satellite navigation signals are distorted due to jamming signals, or satellite signals are tracked using incorrect signals, signal synchronization is lost. A method that blocks the signals that are transmitted to a receiver when these jamming signals are received could be an anti-jamming technique. A method for judging whether it is a jamming signal includes a technique that measures the Jamming to Noise Power Ratio ( $\mathrm{J} / \mathrm{N})$. If $\mathrm{J} / \mathrm{N}$ is above a reference threshold value, the application of jamming is recognized, and the receiver blocks the signals. In this regard, $\mathrm{J} / \mathrm{N}$ detection is performed at AGC. The applied GPS signal power is lower than that of white Gaussian noise. Thus, when a power that is higher than this is received, it can be judged as a jamming environment (Kim 2013).

\subsubsection{DSP Anti-Jamming Technique}

The anti-jamming technique using DSP is represented by the nulling technique, the Spatial Time Adaptive Processing (STAP), the Spatial Frequency Adaptive Processing (SFAP), and the Amplitude Domain Processing (ADP). In this technique, for the satellite navigation signals received
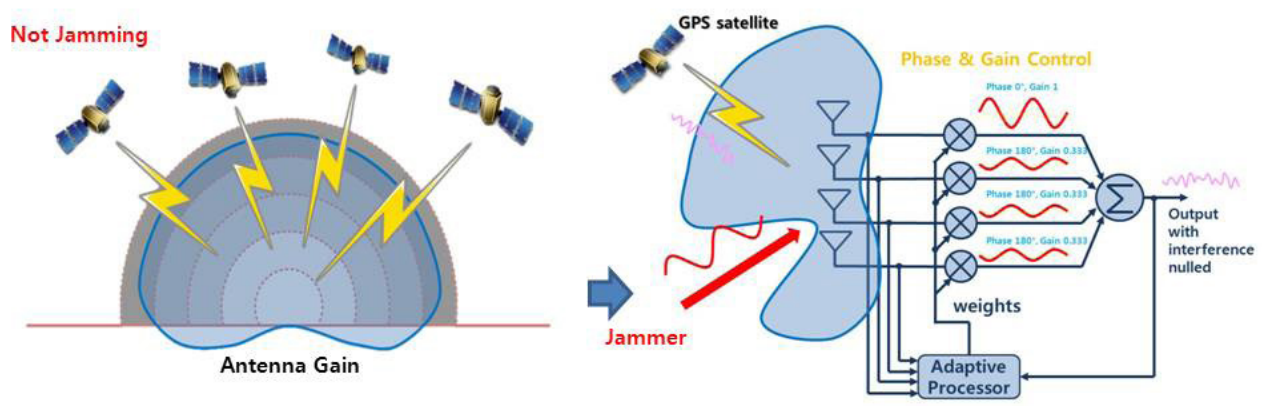

Fig. 3. Null steered array. 


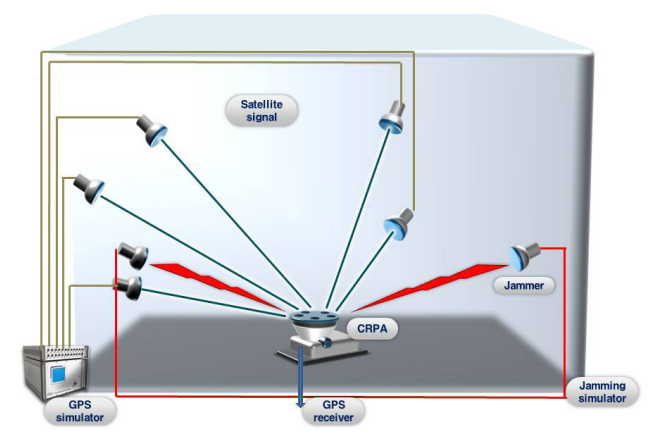

Fig. 4. Configuration of an anechoic chamber test.

from array antenna, jamming and interference signals are eliminated in the frequency time domain and frequency domain using digitized signal samples before demodulation (Gupta \& Moore 2004, Kim \& Iltis 2004).

\subsubsection{Code/Carrier Tracking Loop Technique}

The performance of code/carrier tracking technique is dependent on pre-detection bandwidth. As the bandwidth decreases, the anti-jamming performance and tracking performance are improved, but the dynamic characteristic of the Line Of Sight (LOS) that can receive satellite navigation signals decreases. If an auxiliary system such as Inertial Navigation System (INS) is applied to use velocity information in order to supplement this disadvantage, the bandwidth of the tracking loop can be decreased (Kim 2013).

\subsection{Anti-Jamming Evaluation Technique}

The anti-jamming evaluation technique can be divided into an anechoic chamber test and an outdoor jamming test. For the chamber test, the downscaled simulation test of an actual jamming environment is performed in an environment without external interference; and for the outdoor test, the test is performed by a dynamic test and a static test.

\subsubsection{Anechoic Chamber Test}

Fig. 4 shows a configuration example of an anechoic chamber test. This test evaluation method minimizes external signal interference, enables independent arrangement of satellite signals through a GNSS simulator, and can transmit the same satellite signals at each test. On the other hand, it requires a large chamber, and a lot of manpower and correction time are required when antenna

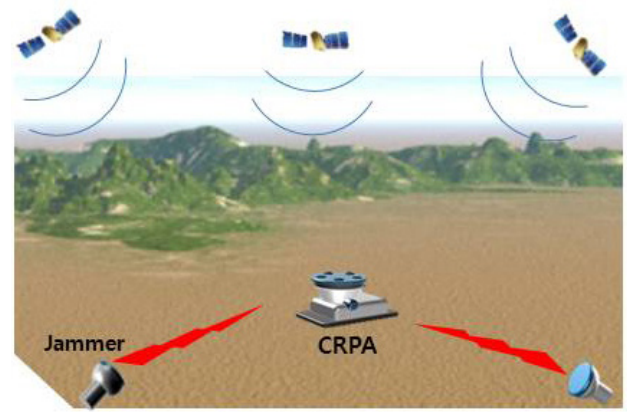

Fig. 5. Configuration of an outdoor test.

and simulator are installed within the chamber (Bae et al. 2010).

\subsubsection{Outdoor Test}

An outdoor test is performed by receiving actual satellite navigation signals, and thus, the test environment is the most similar to an actual jamming environment. However, it affects other equipment and facilities using GNSS signals depending on the radiation of jamming signals; an extensive site needs to be selected when a dynamic test is performed; and ancillary equipment is required. Also, as the arrangement of satellites changes each time, repeated tests at the same condition are unavailable (Bae et al. 2010). Fig. 5 shows a configuration example of an outdoor test.

In this study, to resolve the above-mentioned problems of the chamber test and the outdoor test, an array antenna hood that can inspect the anti-jamming function of an antijamming system without a special tool at the location where the target system for measurement has been constructed was manufactured.

\section{DESIGN AND FABRICATION OF ARRAY- ANTENNA HOOD}

\subsection{Design of an Array Antenna Hood}

To evaluate an anti-jamming system, common radiation of satellite signals and jamming signals is required. The designed hood enables the radiation signals to be applied to the array antenna of CRPA system in the near-field. Fig. 6 shows the conceptual diagram of the hood.

For the hood, the number and arrangement of elements and the shape of the housing are determined depending on the applied anti-jamming system. The hood examines normal operation depending on the coupling strength of the reception array antenna that is applied to an antijamming system, and complements the disadvantages of an 


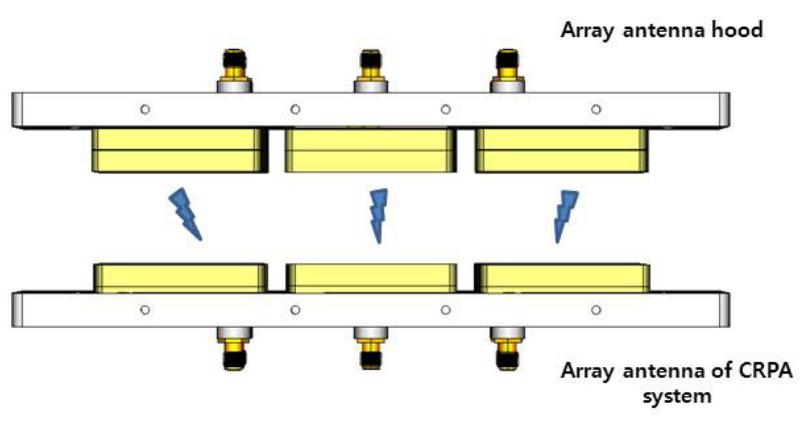

Fig. 6. Conceptual diagram of the hood.

Table 1. Target performance of array antenna hood.

\begin{tabular}{lcl}
\hline \multicolumn{1}{c}{ ITEM } & $\begin{array}{c}\text { Target } \\
\text { performance }\end{array}$ & \multicolumn{1}{c}{ Remarks } \\
\hline Frequency band & GPS L1/L2, & \\
& GLONASS L1 & \\
Element number & 5 \\
VSWR & $<2.0: 1$ & \\
Polarization & RHCP & \\
Mutual coupling & $<-12 \mathrm{~dB}$ & \\
Signal transduction & $>-9 \mathrm{~dB}$ & Same place \\
& & {$[(\mathrm{T} 1-\mathrm{R} 1),(\mathrm{T} 2-\mathrm{R} 2),(\mathrm{T} 3-\mathrm{R} 3),(\mathrm{T} 4-\mathrm{R} 4)$,} \\
& & (T5-R5)] \\
& $<-12 \mathrm{~dB}$ & Non same place \\
& & {$[(\mathrm{T} 1-\mathrm{R} 2),(\mathrm{T} 1-\mathrm{R} 3),(\mathrm{T} 1-\mathrm{R} 4),(\mathrm{T} 1-\mathrm{R} 5) \bullet \bullet \cdot]$} \\
\hline
\end{tabular}

outdoor test and an anechoic chamber test by the near-field radiation of satellite signals and jamming signals.

The array antenna hood plays a role in radiating satellite signals and jamming signals for an anti-jamming system. Accordingly, an important characteristic of the hood is the radiation of satellite signals and jamming signals for an anti-jamming system without the leakage of signals. Thus, it was designed so that signal leakage could be minimized. The target performance of the array antenna hood can be divided into two parts: (1) the performance of the hood and (2) the signal transmission characteristics related with the reception array antenna that is applied to an antijamming system in terms of signal transmission concept. The performance of the hood reflects the concept of array antenna, and thus, the standard of general array antenna was applied. The signal transmission characteristics related with reception array antenna were differently applied depending on the reception array antenna.

For the reception array antenna of the anti-jamming system used in this study, a 5-element array antenna was used; and for the array antenna hood, a 5-element array antenna was also selected. Table 1 summarizes the established target standard, and the operating frequency bands are GPS L1/L2 and GLONASS L1. The normal operation of the reception array antenna of the anti-

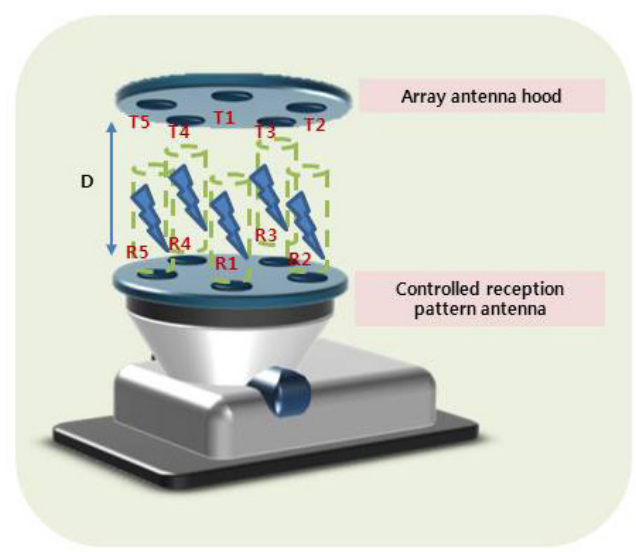

Fig. 7. Position of the anti-jamming system reception array antenna and the hood.

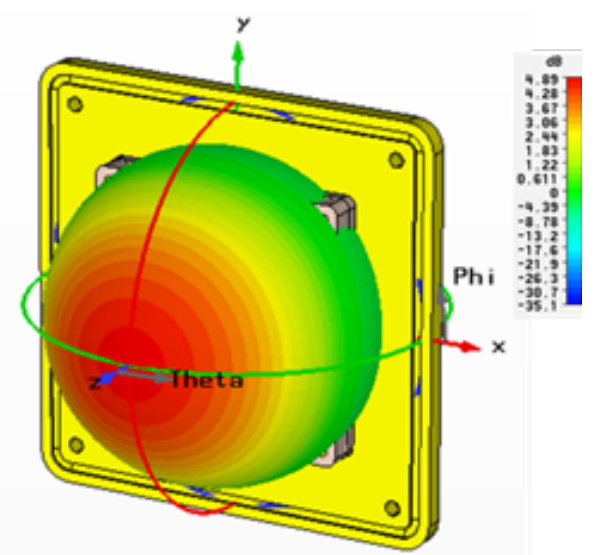

Fig. 8. Radiation pattern of a general patch antenna.

jamming system can be examined by the signal transmission characteristics related with the hood. When a signal is transmitted from the position of each element of the hood, the strength of the signal that is transmitted to the element at the same position of the reception array antenna and the strength of the signal that is transmitted to the element at different position are the signal transmission characteristics. The leakage of the signal should be small for the same position, and the normal operation of each element can be independently examined when the signal transmission to different position is minimized. Fig. 7 shows the conceptual diagram for the position of the elements of the array antenna hood, and Fig. 8 shows the radiation pattern of a general patch antenna. For a patch antenna, the gain is high in the zenith direction. Based on this, signal leakage can be minimized when each element of the hood and the anti-jamming system reception array antenna is designed so that the zenith directions can face each other. For the signal transmission characteristics, the signal transmission characteristics for different position can be determined by 


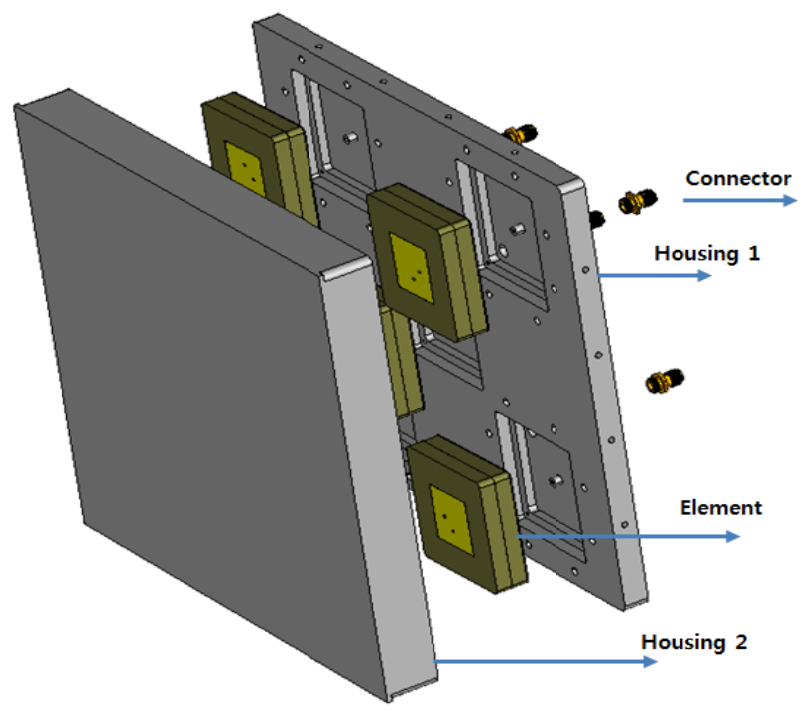

Fig. 9. Configuration of the array antenna hood.

the mutual coupling characteristics of the anti-jamming system reception array antenna. In this study, considering the application size and the arrangement of the elements of the array antenna through a simulation, $-12 \mathrm{~dB}$ was set as the reference. Also, the signal transmission characteristic for the same position was set to below $-9 \mathrm{~dB}$ depending on the isolation characteristics between the hood and the antijamming system reception array antenna.

Fig. 9 shows the configuration of the array antenna hood. It can be divided into the single elements that generate frequency bands, and the housing that protects the single elements and combines an anti-jamming system. Antijamming tests should be enabled while an anti-jamming system is installed at a vehicle. Thus, the housing is designed considering the reception antenna and the vehicle shape of the part at which the reception antenna is installed. The housing requires an input connector that can apply satellite signals and jamming signals. For the material of the housing, policabonate (PC), which does not damage the part where the anti-jamming system reception array antenna is installed and which does not affect the pattern, was used. For the single element, a patch with a laminate structure was used to satisfy multi-bands. As shown in Fig. 7 , the position of the single element was designed so that it could be aligned with the position of the element of the anti-jamming system reception array antenna, in order to minimize the signal leakage.

The array antenna hood plays a role in examining the normal operation of the anti-jamming system reception array antenna and wirelessly radiating satellite signals and jamming signals. Thus, the distance to the anti-jamming system reception array antenna is also an important design

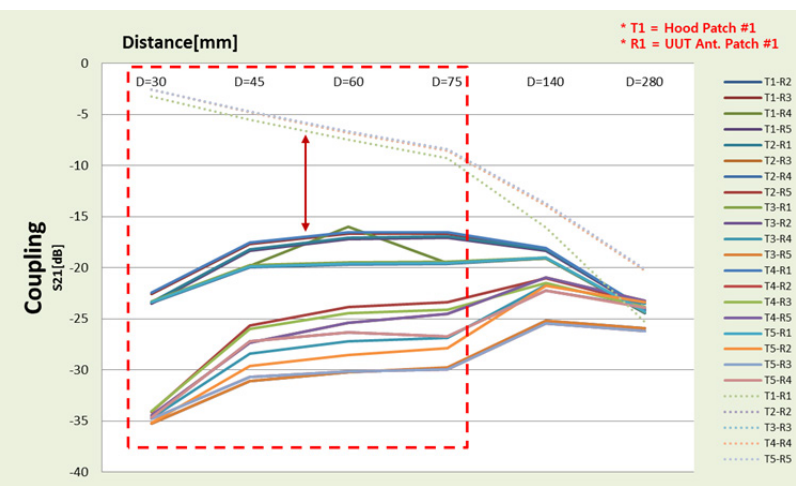

Fig. 10. Simulation of the coupling between the hood and the antijamming system array antenna depending on the distance.

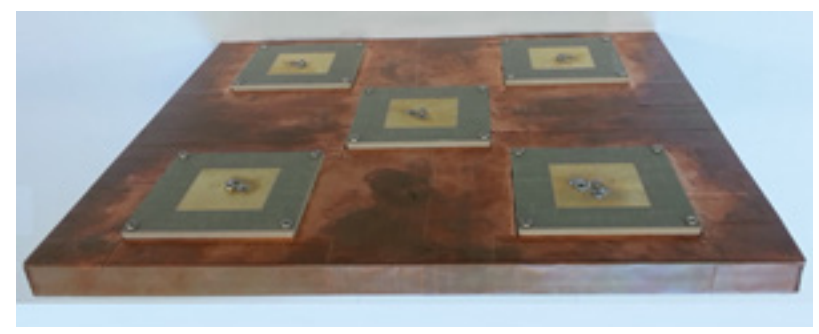

Fig. 11. Manufactured array antenna hood.

factor. For this design, the distance was optimized through a simulation. In the case of the simulation tool, CST MWS was used. Fig. 10 shows the results of the simulation depending on the distance, $\mathrm{D}$, shown in Fig. 7. The $\mathrm{X}$-axis is the distance between the hood and the anti-jamming system reception array antenna, and the Y-axis is the signal transmission characteristic for the corresponding distance. The results of the simulation indicated that as the distance to the antijamming system reception array antenna increased, the loss between the patches at the same position increased and the loss between the patches at different position decreased, which deteriorated the isolation characteristics between the reception antenna and the array antenna hood. Based on this simulation, the distance between the array antenna hood and the anti-jamming system reception array antenna was determined to be within $75 \mathrm{~mm}$, where the results for the same position (dotted lines in Fig. 10) were above $-9 \mathrm{~dB}$, and the results for different position (solid lines in Fig. 10) were below $-12 \mathrm{~dB}$.

\subsection{Manufacture of an Array Antenna Hood}

An array antenna hood was manufactured by reflecting the design content of Section 3.2. Fig. 11 is the photograph of the manufactured array antenna hood. The array antenna hood, where the distance between the array antenna hood and the adaptive array antenna was made to be about 75 


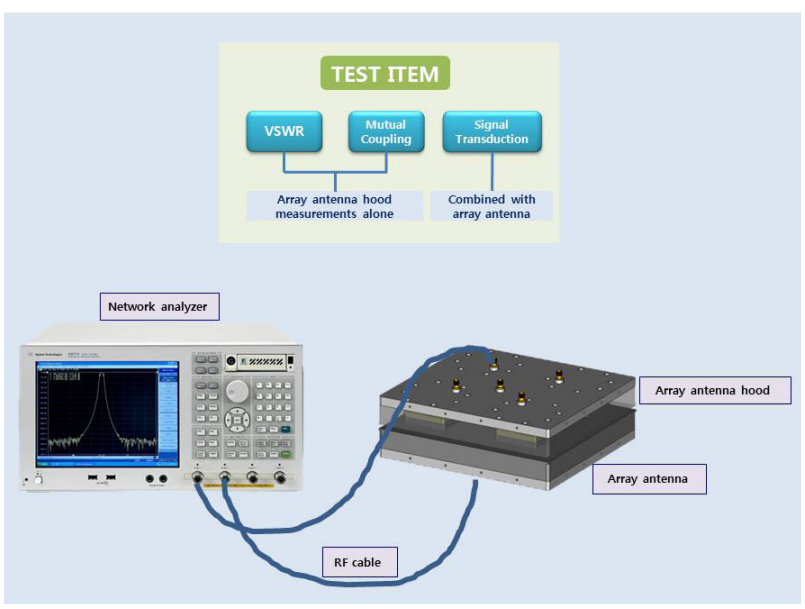

Fig. 12. Measurement item and configuration of the array antenna hood.

Table 2. Signal transduction performance of array antenna hood.

\begin{tabular}{ccrrrrr}
\hline \multirow{2}{*}{ S21 } & \multicolumn{7}{c}{ Array antenna port } \\
\cline { 2 - 7 } & & \multicolumn{1}{c}{ R1 } & \multicolumn{1}{c}{ R2 } & \multicolumn{1}{c}{ R3 } & \multicolumn{1}{c}{ R4 } & \multicolumn{1}{c}{ R5 } \\
\hline \multirow{4}{*}{ HOOD port } & T1 & -5.1 & -28.7 & -15.6 & -31.3 & -27.6 \\
& T2 & -24.9 & -6.5 & -20.0 & -28.3 & -29.7 \\
& T3 & -28.3 & -28.9 & -7.3 & -22.6 & -30.2 \\
& T4 & -28.3 & -28.2 & -29.8 & -6.6 & -33.6 \\
& T5 & -28.4 & -20.5 & -24.1 & -30.2 & -7.0 \\
\hline
\end{tabular}

Table 3. Performance of array antenna hood.

\begin{tabular}{lccl}
\hline \multicolumn{1}{c}{ ITEM } & $\begin{array}{c}\text { Target } \\
\text { performance }\end{array}$ & Result & \multicolumn{1}{c}{ Remarks } \\
\hline VSWR & $<2.0: 1$ & $1.9: 1[\mathrm{Max}]$. & \\
Mutual & $<-12 \mathrm{~dB}$ & $-17 \mathrm{~dB}$ & \\
coupling & & {$[$ Max.] } & \\
Signal & $>-9 \mathrm{~dB}$ & $-7.3 \mathrm{~dB}$ & Same place \\
transduction & & {$[$ Min. $]$} & {$[(\mathrm{T} 1-\mathrm{R} 1),(\mathrm{T} 2-\mathrm{R} 2),(\mathrm{T} 3-\mathrm{R} 3)$,} \\
& & & (T4-R4), (T5-R5)] \\
& $<-12 \mathrm{~dB}$ & $-15.6 \mathrm{~dB}$ & $\begin{array}{l}\text { Non same place } \\
\end{array}$ \\
& & {$[$ Max.] } & [(T1-R2),(T1-R3), (T1-R4), \\
& & (T1-R5)••• $]$ \\
\hline
\end{tabular}

$\mathrm{mm}$, was tested based on the configuration shown in Fig. 12.

To examine the performance of the array antenna hood, the voltage standing wave ratio and the mutual coupling performance were examined using a network analyzer. To examine the signal transmission characteristics, it was combined with the anti-jamming system reception array antenna, and then the signal transmission characteristics were examined. Table 2 summarizes the results of the measurement. For the same position, the minimum value was -7.3 dB; and for different position, the maximum value was $-15.6 \mathrm{~dB}$. The results showed that when a signal was radiated from Patch 1 of the array antenna hood, the attenuation of the signal that arrived at Patch 1 of the antijamming system reception array antenna was small, and the

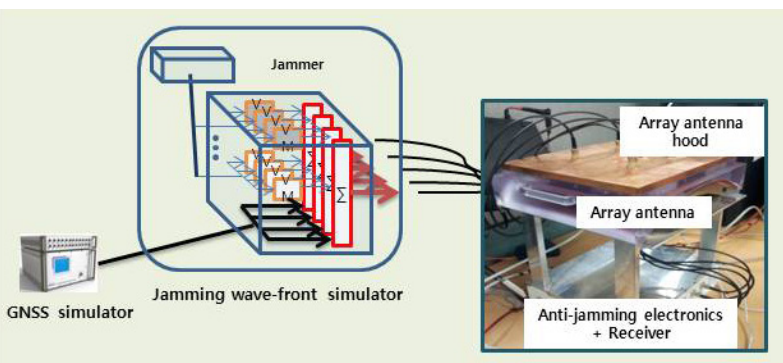

Fig. 13. Configuration of the test of the anti-jamming system function.

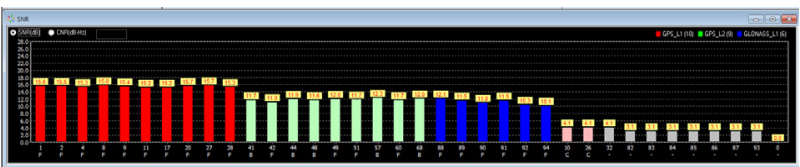

(a) Not jamming signal (only GNSS signal)

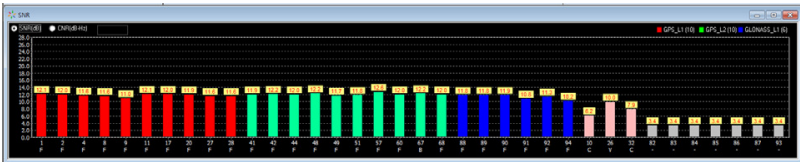

(b) Jamming signal with GNSS signal (GPS L1 jamming)

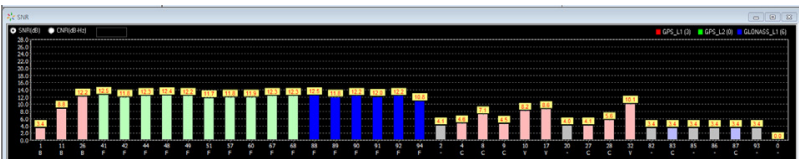

(c) Jamming signal with GNSS signal (GPS L1 no fixed)

Fig. 14. Reception test depending on the changes in the jamming signals.

signal was weakly transmitted to the remaining patches. The remaining four patches also showed similar results.

Table 3 summarizes the results of the signal transmission test for examining the performance of the array antenna hood and the normal operation of the anti-jamming system reception array antenna. A margin of more than about $5 \mathrm{~dB}$ was observed compared to the target performance.

Fig. 13 shows the configuration for examining the function of the anti-jamming system. The jamming wavefront simulator determines the direction of satellite signals and the direction and number of jamming signals by combining the satellite signals of the GNSS simulator and the jamming signals. In this regard, the direction of satellite signals and the direction of jamming signals can be set in specific directions (up to five directions) through the arbitrary adjustment of the strength and phase of the signals.

Fig. 14 shows the results of the reception test depending on the changes in the jamming signals. During the test, the satellite signals were fixed at a strength of $-110 \mathrm{dBm}$ through the GNSS simulator, and the jamming signals were arbitrarily adjusted in the GPS L1 band. In Fig. 14, Picture a 
shows the reception test without jamming signals. Pictures $\mathrm{b}$ and $\mathrm{c}$ show the changes in the reception level depending on the increase in the strength of the jamming signals.

The results of the above test showed that the manufactured array antenna hood could inspect the function of the anti-jamming system as well as could examine the normal operation of the anti-jamming system reception array antenna.

\section{CONCLUSIONS}

In this study, an array antenna hood for examining the normal operation of the reception array antenna of an anti-jamming system and for performing the evaluation test of the system function was manufactured. Based on a test, it was demonstrated that the manufactured array antenna hood could perform the evaluation of the antijamming system function by radiating satellite signals and jamming signals without a spatial restriction. This method can examine the normal operation and function without separating the adaptive array antenna system that is combined with a weapon system, and thus can examine the function of an anti-jamming system without the repeated application of the method that performs evaluation by receiving outdoor satellite signals or the quantitative evaluation method using an expensive anechoic chamber. Therefore, it is expected to be highly useful for weapon systems in the future.

\section{REFERENCES}

Bae, J. S., Kim, J. O., \& Park, H. W. 2010, Anti-Jamming GNSS Receiver Test and Evaluation Program using Embedded Jammer of Aircraft's POD in Dynamic Environment, the 17th GNSS Workshop, Jeju

Gupta, I. J. \& Moore, T. D. 2004, Space-frequency adaptive processing (SFAP) for radio frequency interference mitigation in spread-spectrum receivers, IEEE Transactions on Antennas and Propagation, 52-6, 16111616

Maloney, J. G., Baker, B. N., Acree, J. J., Schultz, J. W., Little, J. A., et al., 2007, Fragmented aperture antenna design of miniaturized GPS CRPA: model and measurements, Antennas and Propagation Society International Symposium, 2007 IEEE, 3784-3787

Kim, K. Y. 2013, Analysis of Anti-Jamming Techniques for Satellite Navigation Systems, J-KICS, 38C-12, 1216-1227

Kim, S. J. \& Iltis, R. A. 2004, STAP for GPS receiver synchronization, IEEE Transactions on Aerospace and Electronic Systems, 40-1, 132-144

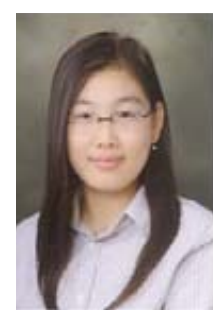

Mi-Suk Kim received the Master's degree in Radio-Wave Engineering from Hanbat National University in 2007. She has worked with Navcours co., ltd. Her research interests include GNSS antenna and anti-jamming antenna.

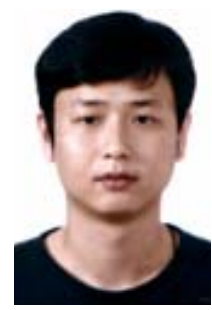

Jeong-Gab Ju received the B.S. and M.S degree in Radio Communication and Engineering from Korea Maritime and Ocean University, 2010, 2012 respectively. He has worked with Navcourse co. ltd. His research interests include GNSS antenna and jamming test equipment.

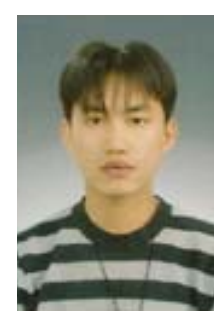

Jong Seong Kim received the Master's degree in Radio-Wave Engineering from Hanbat National University in 2007. He has worked with Navcours co., ltd. His research interests include high quality antenna and jamming test equipment.

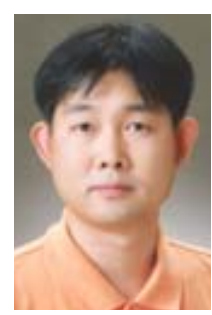

Seok Bo Son received B.S, M.S and Ph.D degree in electronics engineering from Chungnam National University in 1996, 1998 and 2002, respectively. He has worked with Navcours co., ltd. and now is a division director. His current research interests include alternative navigation system, high quality antenna and jamming test equipment.

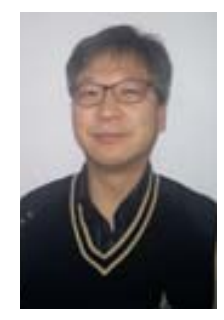

Sang Jun Yun received B.S, M.S and Ph.D degree in electronics engineering from Chungnam National University in 1993, 1995 and 2003, respectively. He has worked with Navcours co., ltd. and now is a R\&D center chief. His current research interests include navigation and timing system design and anti-jamming technique. 